\section{SAT0610 TEMPORAL ARTERY ULTRASOUND IN THE DIAGNOSIS OF GIANT CELL ARTERITIS IN A COHORT WITH ELEVATED CLINICAL IMPRESSION}

P.V. Estrada ${ }^{1}$, D. Reina ${ }^{1}$, V. Navarro ${ }^{1}$, D. Roig ${ }^{1}$, D. Cerdà ${ }^{2}$, V. Torrente ${ }^{1}$, S. Heredia ${ }^{1}, \mathrm{H}$. Corominas ${ }^{1} .{ }^{1}$ Rheumatology; ${ }^{2}$ Hospital Moisès Broggi-Hospital General de L'hospitalet. Consorci Sanitari Integral. Sant Joan Despí, Barcelona, Barcelona, Spain

Background: Giant cell arteritis (GCA) is the most frequent vasculitis in adulthood. The delay in diagnosis sets back treatment and can lead to serious consequences. Diagnosis is complex, and is followed by the classification criteria according to the American College of Rheumatology (ACR). The is an increasing interest on the utility of temporal artery ultrasound (TAUS) as a tool to evaluate inflammation on the vessel wall.

Objectives: to evaluate the utility of TAUS in GCA.

Methods: During 2016, 120 TAUS were carried out in 60 patients with clinical suspicion for GCA. The TAUS was carried to completion by rheumatologist with experience. The symptoms that lead to a TAUS was either one or more of these clinical scenarios: 1) cranial symptoms (recent onset headache, mandibular claudication, visual disturbances) 2) polymyalgic syndrome 3) toxic o febril unspecific syndrome 4) vertebrobasilary (VB) stroke. Demographic and laboratory data were collected, and a follow-up was done to learn the final diagnosis. As for TAUS, the "halo" sign was considered positive if an anechoic image surrounded the vessel was present, and measured $>0,30 \mathrm{~mm}$ in both, longitudinal and transverse cuts. Other more unspecific signs as stenosis or occlusion were also registered. A temporal artery biopsy was performed whenever the physicians considered necessary, based on clinical criteria, every case in no more than 30 days.

Results: Fifty-two percent were women, mean age $76 \pm 7.8$ years old. Mean laboratory parameters: eritrosedimentation rate $85 \pm 41.9 \mathrm{~mm} / \mathrm{h}$, C-reactive protein $77 \pm 80 \mathrm{mg} / \mathrm{L}$, Haemoglobin $11.4 \pm 2.2 \mathrm{~g} / \mathrm{L}$, white blood count $10,228 \pm 3,520$, platelet count $310,603 \pm 123,918$. The symptoms that motivated requesting the TAUS were: cranial symptoms $(62.2 \%)$, toxic, unspecific, febrile syndrome (44\%), polymyalgic syndrome $(30 \%)$, VB stroke $(5 \%)$. A temporal artery biopsy was carried out in $45 \%$ of patients ( $\mathrm{N}=27$ ); it was positive in $40.7 \%$, negative in $40.7 \%$ and unspecific (given it reported an inflammatory histologic pattern, but without the characteristic giant cells) in $18.5 \%$. From all 60 patients in whom a TAUS was performed, $36 \%$ were diagnosed with GCA, based on ACR criteria.

Patients with a final GCA diagnosis $(n=22)$

\begin{tabular}{llc}
\hline Ultrasound & Biopsy & $\%$ \\
\hline Positive & Positive & $40.7 \%$ \\
Positive & Unspecific & $22.7 \%$ \\
Positive & Not done & $9 \%$ \\
Negative & Not done & $9 \%$ \\
Non-conclusive & Negative & $9 \%$ \\
negative & Positive & $9 \%$ \\
\hline
\end{tabular}

The sensibility and specificity for TAUS was $80 \%$ and $94 \%$ respectively, with a posivite predictive value of $88.9 \%$ and a negative predictive value of $89.2 \%$

Conclusions: TAUS is a useful, non-invasive, fast, accessible tool for evaluating temporal arteries with a great diagnostic valu

Disclosure of Interest: None declared

DOI: 10.1136/annrheumdis-2017-eular.6810

\section{SAT0611 INTIMA-MEDIA THICKNESS REFERENCE RANGES DEPICTING HALO SIGN FOR THE DIAGNOSING OF LARGE VESSEL GIANT CELL ARTERITIS BY ULTRASOUND}

M. Milchert, M. Brzosko. Department of Rheumatology Internal Medicine and Geriatrics, Pomeranian Medical University, Szczecin, Poland

Background: Color duplex ultrasonography (CDU) is most promising tool for the assessment of large vessel giant cell arteritis (LV-GCA). There is a need to define ultrasound findings consistent with the diagnosis of GCA.

Objectives: We aimed to score intima-media thickness (IMT) reference ranges for LV-GCA in polish patients.

Methods: 214 patients suspected for GCA and evaluated with CDU were included in the study and followed up for min 9 months. Large vessel CDU, together with arteritis/non-arteritis categorization, were performed before or within 1 week after treatment initiation by a single physician. Vasculitis was defined as hypoechoic, homogenous, increase of IMT with distorted wall structure resulting in no clear intima-media structure, over long distance (not limited only to the place of arterial bifurcations). ROC curves were calculated.

Results: GCA was diagnosed in 81 patients, polymyalgia rheumatica (PMR) in 131 (characteristics - Table 1). Extracranial LV-GCA was diagnosed in 43 patients: axillary vasculitis in 23 patients, common carotid artery (CCA) - 24, subclavian -18 , superficial femoral -11 , brachial (all spreading per continuum from axillary arteritis) -8 . In 83 remaining patients other diagnosis was confirmed, and they served as non-GCA/PMR controls. Mean IMT in LV-GCA was significantly higher versus controls and isolated PMR (Fig. 1). IMT in GCA was not significantly influenced by gender, hypertension and smoking in contrast with IMT in controls. Proposed cut off values for IMT depicting vasculitis in GCA patients are presented in Table 2. $100 \%$ specificity for vasculitis (vs GCA without large vessel vasculitis) was reached with axillary IMT of $1.06 \mathrm{~mm}$ ( $62 \%$ sens.), subclavian $-1.35 \mathrm{~mm}(38 \%$ sens.), superficial femoral $-1.55 \mathrm{~mm}(60 \%$ sens.), CCA $-1.27 \mathrm{~mm}(22 \%$ sens.)

Table 1. Patient characteristics

\begin{tabular}{|c|c|c|c|c|}
\hline & $\begin{array}{c}\mathrm{GCA} \\
(\mathrm{N}=81)\end{array}$ & $\begin{array}{l}\text { Isolated PMR } \\
(\mathrm{N}=50)\end{array}$ & $\begin{array}{c}\text { Non-GCA/PMR controls } \\
(\mathrm{N}=83)\end{array}$ & $\mathrm{p}$ \\
\hline Female & $53(65 \%)$ & $37(74 \%)$ & $54(65 \%)$ & 0.512 \\
\hline Age $($ mean $\pm S D ;$ min-max & $73^{\star} \pm 9 ; 55-95$ & $69 \pm 9 ; 52-87$ & $65 \pm 10 ; 44-89$ & 0.001 \\
\hline Hypertension & $53(65 \%)$ & $31(62 \%)$ & $44(53 \%)$ & 0.251 \\
\hline Smoking & $36(44 \%)$ & $14(28 \%)$ & $29(35 \%)$ & 0.148 \\
\hline Diabetes & $13(16 \%)$ & $6(12 \%)$ & $15(18 \%)$ & 0.649 \\
\hline Hypercholesterolemia** & $25 / 66(38 \%)$ & 12/33 (36\%) & $19 / 38(50 \%)$ & 0.400 \\
\hline Arterial calcifications ${ }^{\star \star \star}$ & $34(42 \%)$ & $11(22 \%)^{*}$ & $33(40 \%)$ & 0.058 \\
\hline Upper limbs claudication & $4(4.9 \%)$ & $0(0.0 \%)$ & $1(1.2 \%)$ & 0.131 \\
\hline
\end{tabular}

*Significant findings $(p<0.05)$. **Assessed in 137 patients; *** Of medium to high grade.

Table 2. Cut off values for IMT depicting vasculitis in GCA patients

\begin{tabular}{lcccc}
\hline Artery & IMT $(\mathrm{mm})$ & AUC & Sens. $(\%)$ & Spec. $(\%)$ \\
\hline Arteries classified as vasculitis vs & GCA & without & large vessel vasculitis & \\
$\quad$ Axillary & 0.82 & 0.959 & 85.7 & 92.7 \\
Subclavian & 0.84 & 0.954 & 87.5 & 96.8 \\
Superficial femoral & 0.97 & 0.945 & 90.0 & 96.3 \\
Common carotid & 0.96 & 0.877 & 72.2 & 93.7 \\
Arteries classified as vasculitis vs controls & & & \\
$\quad$ Axillary & 0.81 & 0.969 & 87.0 & 93.7 \\
Subclavian & 0.66 & 0.974 & 100 & 83.7 \\
Superficial femoral & 0.92 & 0.958 & 90.9 & 96.6 \\
Common carotid & 0.73 & 0.910 & 91.7 & 79.0 \\
\hline
\end{tabular}

Maximal IMT value from bilateral CDU measurements was chosen. $A U C=$ area under the curve.

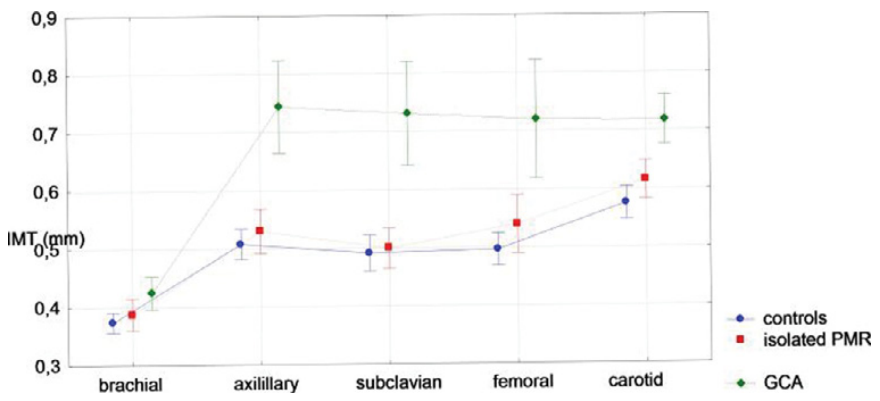

Figure 1. Mean IMT in different arteries in GCA, isolated PMR and controls.

Conclusions: We demonstrated that cut off values may discriminate between GCA and its mimics as well as between presence and lack of vasculitis in different arteries in GCA

References:

[1] Milchert M, Diamantopoulos A, Brzosko M. Atlas of ultrasound application in large vessel arteritis: giant cell arteritis and Takayasu arteritis. Wydawnictwo Pomorskiej Akademii Medycznej, Szczecin, 2016.

Disclosure of Interest: None declared

DOI: 10.1136/annrheumdis-2017-eular.4139

\section{SAT0612 THE USE OF 18F-FDG-PET IN THE DIAGNOSIS OF POLYMYALGIA RHEUMATICA (PMR) - A PROSPECTIVE STUDY OF 99 PATIENTS SUSPECTED OF PMR}

L. Henckaerts ${ }^{1}$, O. Gheysens ${ }^{2}$, S. Vanderschueren ${ }^{1}$, K. Goffin ${ }^{2}$,

D. Blockmans ${ }^{1} .{ }^{1}$ General Internal Medicine; ${ }^{2}$ Nuclear Medicine Department,

University Hospital Gasthuisberg, Leuven, Belgium, Leuven, Belgium

Background: Previous studies have shown that the majority of patients with polymyalgia rheumatica (PMR) have increased fluorodeoxyglucose (FDG)-uptake around the shoulders, hips and processes of the cervical and lumbar spine on positron emission tomography (PET) (1). The specificity of these findings for PMR is not known.

Objectives: To determine the specificity and sensitivity of FDG-PET findings for the diagnosis of PMR

Methods: A prospective monocentric study in a tertiary care centre. All patients underwent FDG-PET scanning before treatment with glucocorticoids was started. The clinical suspicion of PMR was quantified by the treating physician on a scale from 1 to 5 . FDG-uptake was scored visually in 12 articular regions (cervical spinous processes, lumbar spinous processes, left and right sternoclavicular joint, left and right ischial tuberosity, left and right greater trochanter, left and right hip and left and right shoulder) (score 0-2) and a total skeletal score was calculated reflecting the FDG-uptake in these 12 articular regions. ROC analysis was performed to determine the optimal clinical and total skeletal score for diagnosing PMR. The golden standard for a diagnosis of PMR was the judgment of an experienced clinician after at least six months of follow-up.

Results: 99 consecutive patients with a possible clicinal diagnosis of PMR were included in this study. Sixty-seven patients were finally diagnosed with PMR while 32 patients got another diagnosis. A clinical score of 4 or more had a sensitivity 